\title{
Study of implicit delay fractional differential equations under anti-periodic boundary conditions
}

\author{
Arshad Ali ${ }^{1}$, Kamal Shah' ${ }^{1}$ and Thabet Abdeljawad ${ }^{2,3,4^{*}}$
}

\section{"Correspondence:}

tabdeljawad@psu.edu.sa

${ }^{2}$ Department of Mathematics and

General Sciences, Prince Sultan

University, Riyadh, Saudi Arabia

${ }^{3}$ Department of Medical Research,

China Medical University, Taichung,

Taiwan

Full list of author information is

available at the end of the article

\begin{abstract}
This research work is related to studying a class of special type delay implicit fractional order differential equations under anti-periodic boundary conditions. With the help of classical fixed point theory due to Schauder and Banach, we derive some results about the existence of at least one solution. Further, we also study some results including Hyers-Ulam, generalized Hyers-Ulam, Hyers-Ulam Rassias, and generalized Hyers-Ulam-Rassias stability. We provide some test problems for illustrating our analysis.
\end{abstract}

Keywords: Pantograph differential equation; Existence theory; Hyers-Ulam stability

\section{Introduction}

Differential equations have numerous of applications in many applied fields of sciences. Due to these applications, the class of differential equations has remained an interesting area of research. The fractional order derivative is a generalization of the classical derivative, which has been proved to be a strong tool for modeling of many physical, biological, and evolutionary problems. In the recent times this has been the hottest and most interesting area of research in mathematics as well as in other scientific and engineering courses. For some historical and recent work, we refer the readers to [1-9]. A comprehensive study in the form of a book has been given by Podlubny [10].

In previous years, the study of nonlinear differential and integral equations has received much attention from mathematicians due to a wide range of their applications. Since using integer order differential operators for modeling various dynamical systems, the hereditary process and memory description cannot be well explained in many situations. Therefore, researchers are applying the fractional differential operators to describe memory and hereditary processes in a more accurate way. This fact motivated researchers to take interest in fractional order differential equations. Various aspects of fractional calculus, such as qualitative theory, stability analysis, optimization, and numerical analysis, have been investigated. In this regard a lot of research work can be found in the literature about existence theory. We refer the readers to [11-14]. On the other hand, the area devoted

(c) The Author(s) 2020. This article is licensed under a Creative Commons Attribution 4.0 International License, which permits use sharing, adaptation, distribution and reproduction in any medium or format, as long as you give appropriate credit to the original author(s) and the source, provide a link to the Creative Commons licence, and indicate if changes were made. The images or other third party material in this article are included in the article's Creative Commons licence, unless indicated otherwise in a credit line to the material. If material is not included in the article's Creative Commons licence and your intended use is not permitted by statutory regulation or exceeds the permitted use, you will need to obtain permission directly from the copyright holder. To view a copy of this licence, visit http://creativecommons.org/licenses/by/4.0/. 
to establishing a procedure for numerical solutions has been investigated very well. See [15-18] and the references therein.

It is necessary for numerical procedure to be stable to produce good results, which is highly acceptable in applications. For this purpose stability analysis is used. This is an important aspect of qualitative analysis. Various kinds of stability, including exponential, Mittag-Leffler, and Lyapunov stability, have been evaluated for a number of problems. In the last few years the mentioned stabilities have been upgraded for linear and nonlinear fractional order differential equations and their systems (for details, see [19-21]). Establishing these stabilities for nonlinear systems has merits and de-merits in constructions. Some of them need a pre-defined Lyapunov function, which often is very difficult and time consuming to construct on trail basis. On the other hand, the exponential and Mittag-Leffler stability involving exponential functions often create difficulties in treating during numerical analysis of problems. In 1940-41, Ulam and Hyers introduced the concept of Hyers-Ulam stability. This concept of stability was initially used for functional equations; for details, we refer to [22, 23]. Onward the said stability was further modified to a more general form by other researchers for functional equations, ordinary differential equations. Some very fruitful results have been formed in this regard, which can be traced in [24-26] and the references therein. In the last two decades the said stability theory has been considered very well for fractional order differential equations and their systems, see $[27,28]$.

The delay differential equations constitute an important class of differential equations. Such equations emphasize the waste analysis of full nonlinear equations or systems in biology and physics, as well as in other applied fields. Among delay differential equations, the pantograph type delay differential equation is a prominent type. Such type of delay differential equations has proportional delay terms. Such type of delay differential equations has applications in electro-dynamic, quantum mechanics, etc. [29]. Therefore, keeping in mind the applications, researchers are devoted to studying different aspects like existence theory and numerical analysis of the mentioned class of differential equations. See for detail [30]. The authors [31] in 2013 studied the following pantograph fractional order differential equation with $t \in[0, T]$ :

$$
\begin{aligned}
& { }_{0}^{C} D_{t}^{\alpha} z(t)=f(t, z(t), z(\lambda t)), \\
& z(0)=z_{0}, \quad z_{0} \in R,
\end{aligned}
$$

where $0<\alpha \leq 1,0<\lambda<1$, and $f:[0, T] \times R^{2} \rightarrow R$. They developed the existence theory for the aforesaid equation by using fixed point theory. Very recently the authors in [32] established qualitative theory for a coupled system of delay fractional order differential equations by using hybrid fixed point theory.

Motivated by the above-mentioned work, in this research article we consider the following class of pantograph implicit fractional order differential equations under anti-periodic boundary conditions:

$$
\left\{\begin{array}{l}
{ }_{0}^{C} D_{t}^{\alpha} z(t)=f\left(t, z(t), z(\lambda t),{ }_{0}^{C} D_{t}^{\alpha} z(t)\right), \quad t \in[0, T], 2<\alpha \leq 3, \\
z(0)=-z(T), \quad{ }_{0}^{C} D_{t}^{p} z(0)=-{ }_{0}^{C} D_{t}^{p} z(T), \quad{ }_{0}^{C} D_{t}^{q} z(0)=-{ }_{0}^{C} D_{t}^{q} z(T),
\end{array}\right.
$$


where $0<\lambda<1,0<p<1,1<q<2$, and $f:[0, T] \times R^{3} \rightarrow R$ is a continuous function, ${ }_{0}^{C} D_{t}^{\alpha}$ stands for a Caputo derivative of order $2<\alpha \leq 3$. We investigate qualitative theory as well as different kinds of stability including Hyers-Ulam stability, generalized Hyers-Ulam stability, Hyers-Ulam-Rassias stability, and generalized Hyers-Ulam-Rassias stability for the considered problem. For qualitative theory we utilize the usual fixed point theorem due to Schauder and Banach, while for stability theory nonlinear functional analysis is used. Finally, this work is strengthened by providing examples and short conclusion.

\section{Preliminaries}

The space $\mathcal{M}=C([0, T])$ is a Banach space with respect to the norm defined by

$$
\|z\|_{\mathcal{M}}=\max _{t \in[0, T]}\{|z(t)|: t \in[0, T]\} .
$$

Definition 1 ([33]) Integral of fractional order for the function $z \in L^{1}\left([0, T], R^{+}\right)$of order $\alpha \in R^{+}$is recalled as

$$
{ }_{0} I_{t}^{\alpha} z(t)=\int_{0}^{t} \frac{(t-s)^{\alpha-1}}{\Gamma(\alpha)} z(s) d s
$$

such that the integral on right-hand sides is convergent.

Definition 2 ([33]) Caputo fractional order derivative of a function $z$ on interval $[0, T]$, can be defined as

$$
{ }_{0}^{C} D_{t}^{\alpha} z(t)=\frac{1}{\Gamma(n-\alpha)} \int_{0}^{t}(t-s)^{n-\alpha-1} z^{(n)}(s) d s,
$$

where $n=[\alpha]+1$.

Lemma 1 ([34]) If $\alpha>0$, the given result holds

$$
{ }_{0} I_{t}^{\alpha}\left({ }_{0}^{C} D_{t}^{\alpha} z(t)\right)=z(t)-\sum_{i=0}^{n-1} c_{i} t^{i}, \quad \text { where } n=[\alpha]+1, c_{i} \in R
$$

Definition 3 ([35]) Problem (1) is Hyers-Ulam stable if there exists a real number $\mathrm{C}_{f}>0$ such that, for $\epsilon>0$ and for any solution $\bar{z} \in \mathcal{M}$ of the inequality

$$
\left|{ }_{0}^{C} D_{t}^{\alpha} \bar{z}(t)-f\left(t, \bar{z}(t), \bar{z}(\lambda t),{ }_{0}^{C} D_{t}^{\alpha} \bar{z}(t)\right)\right| \leq \epsilon, \quad \forall t \in[0, T],
$$

there is the unique solution $z \in \mathcal{M}$ of problem (1) such that

$$
|\bar{z}(t)-z(t)| \leq \mathrm{C}_{f} \epsilon, \quad \forall t \in[0, T]
$$

Definition 4 ([35]) Problem (1) is generalized Hyers-Ulam stable if there exists $\zeta \in$ $C\left(R^{+}, R^{+}\right), \zeta(0)=0$ such that, for any solution $\bar{z} \in \mathcal{M}$ of the inequality (5), there is the unique solution $z \in \mathcal{M}$ of problem (1) such that

$$
|\bar{z}(t)-z(t)| \leq \zeta(\epsilon), \quad \forall t \in[0, T] .
$$


Definition 5 ([35]) Problem (1) is Hyers-Ulam-Rassias stable with respect to $\xi \in$ $C\left([0, T], R^{+}\right)$if there exists a real number $C_{f}>0$ such that, for $\epsilon>0$ and for any solution $z \in \mathcal{M}$ of the inequality

$$
\left|{ }_{0}^{C} D_{t}^{\alpha} \bar{z}(t)-f\left(t, \bar{z}(t), \bar{z}(\lambda t),{ }_{0}^{C} D_{t}^{\alpha} \bar{z}(t)\right)\right| \leq \xi(t) \epsilon, \quad \forall t \in[0, T]
$$

there exists the unique solution $z \in \mathcal{M}$ of problem (1) such that

$$
|\bar{z}(t)-z(t)| \leq \mathrm{C}_{f} \epsilon \xi(t), \quad \forall t \in[0, T]
$$

Definition 6 ([35]) Problem (1) will be generalized Hyers-Ulam-Rassias stable with respect to $\xi \in \mathcal{M}$ if there is $\mathrm{C}_{f}>0$ such that, for any solution $\bar{z} \in \mathcal{M}$ of the inequality (6), there exists the unique solution $z \in \mathcal{M}$ of problem (1) such that

$$
|\bar{z}(t)-z(t)| \leq \mathrm{C}_{f} \xi(t), \quad \forall t \in[0, T] .
$$

Remark 1 A function $\bar{z} \in \mathcal{M}$ is a solution of (5) if there is a function $\vartheta(t) \in \mathcal{M}$ (dependent on $\bar{z}$ ) such that

(i) $|\vartheta(t)| \leq \epsilon \forall t \in[0, T]$;

(ii) ${ }_{0}^{C} D_{t}^{\alpha} \bar{z}(t)=f\left(t, \bar{z}(t), \bar{z}(\lambda t),{ }_{0}^{C} D_{t}^{\alpha} \bar{z}(t)\right)+\vartheta(t), \forall t \in[0, T]$.

Remark 2 A function $\bar{z} \in \mathcal{M}$ is a solution of (6) if there is a function $\vartheta(t) \in C([0, T], R)$ (dependent on $\bar{z}$ ) such that

(i) $|\vartheta(t)| \leq \epsilon \xi(t) \forall t \in[0, T]$;

(ii) ${ }_{0}^{C} D_{t}^{\alpha} \bar{z}(t)=f\left(t, \bar{z}(t), \bar{z}(\lambda t),{ }_{0}^{C} D_{t}^{\alpha} \bar{z}(t)\right)+\vartheta(t), \forall t \in[0, T]$.

\section{Existence results}

Theorem 1 Let $y \in C([0, T], R)$, then the equivalent integral equation of the following problem

$$
\begin{cases}{ }_{0}^{C} D_{t}^{\alpha} z(t)=y(t), & \text { for } t \in[0, T], 2<\alpha \leq 3, \\ z(0)=-z(T), & { }_{0}^{C} D_{t}^{p} z(0)=-{ }_{0}^{C} D_{t}^{p} z(T), \quad{ }_{0}^{C} D_{t}^{q} z(0)=-{ }_{0}^{c} D_{t}^{q} z(T)\end{cases}
$$

is given by

$$
z(t)=\int_{0}^{T} \mathcal{W}(t, s) y(s) d s
$$

while the Green's function $\mathcal{W}(t, s)$ is expressed as

$$
\mathcal{W}(t, s)=\left\{\begin{array}{cc}
\frac{(t-s)^{\alpha-1}-\frac{1}{2}(T-s)^{\alpha-1}}{\Gamma(\alpha)}+\frac{\Gamma(2-p)(T-2 t)(T-s)^{\alpha-p-1}}{2 \Gamma(\alpha-p) T^{1-p}} & \\
-\frac{\left[p T^{2}-4 T t+2(2-p) t^{2} \Gamma(3-q)(T-s)^{\alpha-q-1}\right]}{4(2-p) \Gamma(\alpha-p) T^{2}-q}, & 0 \leq s \leq t \leq T, \\
\frac{(T-s)^{\alpha-1}}{2 \Gamma(\alpha)}+\frac{\Gamma(2-p)(T-2 t)(T-s)^{\alpha-p-1}}{2 \Gamma(\alpha-p) T^{1-p}} & \\
-\frac{\left[p T^{2}-4 T t+2(2-p) t^{2} \Gamma(3-q)(T-s)^{\alpha-q-1}\right]}{4(2-p) \Gamma(\alpha-p) T^{2-q}}, & 0 \leq t \leq s \leq T .
\end{array}\right.
$$


Proof Let $z$ be a solution of (9), then by Lemma 1 there exist some real constants $c_{0}, c_{1}, c_{2}$ such that

$$
z(t)={ }_{0} I_{t}^{\alpha} y(t)-c_{0}-c_{1} t-c_{2} t^{2}=\frac{1}{\Gamma(\alpha)} \int_{0}^{t}(t-s)^{\alpha-1} y(s)-c_{0}-c_{1} t-c_{2} t^{2}
$$

Using the results ${ }_{0}^{C} D_{t}^{p} b=0$ ( $b$ is constant), ${ }_{0}^{C} D_{t}^{p} t=\frac{t^{1-p}}{\Gamma(2-p)},{ }_{0}^{C} D_{t}^{p} t^{2}=\frac{2 t^{2-p}}{\Gamma(3-p)}$, and ${ }_{0}^{C} D_{t 0}^{p}{ }_{t}^{\alpha} y(t)=$ ${ }_{0} I_{t}^{\alpha-p} y(t)$, we get

$$
{ }_{0}^{C} D_{t}^{p} z(t)=\frac{1}{\Gamma(\alpha-p)} \int_{0}^{t}(t-s)^{\alpha-p-1} y(s) d s-c_{1} \frac{t^{1-p}}{\Gamma(2-p)}-c_{2} \frac{2 t^{2-p}}{\Gamma(3-p)} .
$$

In view of ${ }_{0}^{C} D_{t}^{q} t=0(1<q<2)$ and ${ }_{0}^{C} D_{t}^{q} t^{2}=\frac{2 t^{2-q}}{\Gamma(3-q)}$, we get

$$
{ }_{0}^{C} D_{t}^{q} z(t)=\frac{1}{\Gamma(\alpha-q)} \int_{0}^{t}(t-s)^{\alpha-q-1} y(s) d s-c_{2} \frac{2 t^{2-q}}{\Gamma(3-q)} .
$$

Applying the boundary conditions $z(0)=-z(T),{ }_{0}^{C} D_{t}^{p} z(0)={ }_{-}^{C} D_{t}^{p} z(T),{ }_{0}^{C} D_{t}^{q} z(0)=$ ${ }_{0}^{C} D_{t}^{q} z(T)$, we find that

$$
\begin{aligned}
c_{0}= & \frac{1}{2 \Gamma(\alpha)} \int_{0}^{T}(T-s)^{\alpha-1} y(s) d s-\frac{\Gamma(2-p) T^{p}}{2 \Gamma(\alpha-p)} \int_{0}^{T}(T-s)^{\alpha-p-1} y(s) d s \\
& +\frac{p \Gamma(3-q) T^{q}}{4(2-p) \Gamma(\alpha-q)} \int_{0}^{T}(T-s)^{\alpha-q-1} y(s) d s, \\
c_{1}= & \frac{\Gamma(2-p)}{\Gamma(\alpha-p) T^{1-p}} \int_{0}^{T}(T-s)^{\alpha-p-1} y(s) d s \\
& -\frac{\Gamma(3-q)}{(2-p) \Gamma(\alpha-q) T^{1-q}} \int_{0}^{T}(T-s)^{\alpha-q-1} y(s) d s, \\
c_{2}= & \frac{\Gamma(3-q)}{2 \Gamma(\alpha-q) T^{2-q}} \int_{0}^{T}(T-s)^{\alpha-q-1} y(s) d s .
\end{aligned}
$$

Substituting the values of $c_{0}, c_{1}$, and $c_{2}$ in (12), one gets the following result:

$$
\begin{aligned}
z(t)= & \frac{1}{\Gamma(\alpha)} \int_{0}^{t}(t-s)^{\alpha-1} y(s) d s-\frac{1}{2 \Gamma(\alpha)} \int_{0}^{T}(T-s)^{\alpha-1} y(s) d s \\
& +\frac{\Gamma(2-p)(T-2 t)}{2 \Gamma(\alpha-p) T^{1-p}} \int_{0}^{T}(T-s)^{\alpha-p-1} y(s) d s \\
& -\frac{\left(p T^{2}-4 T t+2(2-p) t^{2}\right) \Gamma(3-q)}{4(2-p) \Gamma(\alpha-q) T^{2-q}} \int_{0}^{T}(T-s)^{\alpha-q-1} y(s) d s \\
= & \int_{0}^{T} \mathcal{W}(t, s) y(s) d s .
\end{aligned}
$$

Corollary 1 Problem (1) has the following solution:

$$
z(t)=\int_{0}^{T} \mathcal{W}(t, s) f\left(s, z(s), z(\lambda s),{ }_{0}^{C} D_{t}^{\alpha} z(s)\right) d s .
$$

Lemma 2 The function $\mathcal{W}(t, s)$ in (11) obeys the given relations: 
$\left(\mathscr{P}_{1}\right) \mathcal{W}(t, s)$ is continuous over $[0, T]$;

$\left(\mathscr{P}_{2}\right) \max _{t \in[0, T]} \int_{0}^{T} \mathcal{W}(t, s) d s \leq \frac{T^{\alpha}}{\Gamma(\alpha+1)}+\frac{\Gamma(2-p) T^{\alpha}}{2 \Gamma(\alpha-p+1)}+\frac{(p+2) \Gamma(3-q) T^{\alpha}}{2(2-p) \Gamma(\alpha-q+1)}$.

For convenience, we use the notion

$$
\varpi=\frac{T^{\alpha}}{\Gamma(\alpha+1)}+\frac{\Gamma(2-p) T^{\alpha}}{2 \Gamma(\alpha-p+1)}+\frac{(p+2) \Gamma(3-q) T^{\alpha}}{2(2-p) \Gamma(\alpha-q+1)} .
$$

Proof Proof of $\left(\mathscr{P}_{1}\right)$ is obvious. To derive $\left(\mathscr{P}_{2}\right)$, we have

$$
\begin{aligned}
\max _{t \in[0, T]} \int_{0}^{T} \mathcal{W}(t, s) d s= & \max _{t \in[0, T]}\left(\frac{1}{\Gamma(\alpha)} \int_{0}^{t}(t-s)^{\alpha-1} d s-\frac{1}{2 \Gamma(\alpha)} \int_{0}^{T}(T-s)^{\alpha-1} d s\right. \\
& +\frac{\Gamma(2-p)(T-2 t)}{2 \Gamma(\alpha-p) T^{1-p}} \int_{0}^{T}(T-s)^{\alpha-p-1} d s \\
& \left.-\frac{\left[p T^{2}-4 T t+2(2-p) t^{2}\right] \Gamma(3-q)}{4(2-p) \Gamma(\alpha-q) T^{2-q}} \int_{0}^{T}(T-s)^{\alpha-q-1} d s\right) \\
\leq & \max _{t \in[0, T]}\left(\frac{T^{\alpha}}{\Gamma(\alpha+1)}+\frac{\Gamma(2-p) T^{\alpha-p+1}}{2 \Gamma(\alpha-p+1) T^{1-p}}\right. \\
& \left.-\frac{\left[p T^{2}-4 T t+2(2-p) t^{2}\right] \Gamma(3-q) T^{\alpha-q}}{4(2-p) \Gamma(\alpha-q+1) T^{2-q}}\right) \\
\leq & \frac{T^{\alpha}}{\Gamma(\alpha+1)}+\frac{\Gamma(2-p) T^{\alpha}}{2 \Gamma(\alpha-p+1)}+\frac{(p+2) \Gamma(3-q) T^{\alpha}}{2(2-p) \Gamma(\alpha-q+1)} .
\end{aligned}
$$

Hence we have

$$
\max _{t \in[0, T]} \int_{0}^{T} \mathcal{W}(t, s) d s \leq \frac{T^{\alpha}}{\Gamma(\alpha+1)}+\frac{\Gamma(2-p) T^{\alpha}}{2 \Gamma(\alpha-p+1)}+\frac{(p+2) \Gamma(3-q) T^{\alpha}}{2(2-p) \Gamma(\alpha-q+1)} .
$$

To go ahead, we need the following conditions to hold:

$\left(F_{1}\right)$ For $t \in[0, T]$, we have three constants $0<K_{f}<1$ and $L_{f}>0$, with

$$
|f(t, u, v, w)-f(t, \bar{u}, \bar{v}, \bar{w})| \leq L_{f}(|u-\bar{u}|+|v-\bar{v}|)+K_{f}|w-\bar{w}|
$$

for $u, v, w \in \mathcal{M}$.

$\left(F_{2}\right)$ For $t \in[0, T]$, there exist $\theta_{0}, \theta_{1}, \theta_{2} \in \mathcal{M}$ such that

$$
\begin{aligned}
|f(t, u(t), v(t), w(t))| \leq & \theta_{0}(t)+\theta_{1}(t)[|u(t)|+|v(t)|] \\
& +\theta_{2}(t)|w(t)| \quad \text { for } u, v, w \in \mathcal{M},
\end{aligned}
$$

with $\theta_{0}^{*}=\sup _{t \in[0, T]} \theta_{0}(t), \theta_{1}^{*}=\sup _{t \in[0, T]} \theta_{1}(t), \theta_{2}^{*}=\sup _{t \in[0, T]} \theta_{2}(t)<1$.

We define an operator $\mathcal{N}: \mathcal{M} \rightarrow \mathcal{M}$ as

$$
\mathcal{N}(z)(t)=\int_{0}^{T} \mathcal{W}(t, s) \beta_{z}(s) d s
$$

where $\beta_{z}(t) \in C([0, T], R)$ such that $\beta_{z}(t)=f\left(t, z(t), z(\lambda t),{ }_{0}^{C} D_{t}^{\alpha} z(t)\right)$.

Theorem 2 The operator $\mathcal{N}: \mathcal{M} \rightarrow \mathcal{M}$ defined in (14) is completely continuous. 
Proof The continuity of $f, \mathcal{W}(t, s)$ implies the continuity of operator $\mathcal{N}$. Let $\mathbb{B} \subset \mathcal{M}$ be a bounded set such that $\mathbb{B}=\left\{z \in \mathcal{M}:\|z\|_{\mathcal{M}} \leq r\right\}$. Let $z \in \mathbb{B}$. Then

$$
\begin{aligned}
|\mathcal{N} z(t)| & =\left|\int_{0}^{T} \mathcal{W}(t, s) \beta_{z}(s) d s\right| \\
& \leq \int_{0}^{T}|\mathcal{W}(t, s)|\left|\beta_{z}(s)\right| d s .
\end{aligned}
$$

By assumption $\left(F_{2}\right)$, we have

$$
\begin{aligned}
\left|\beta_{z}(t)\right| & =\left|f\left(t, z(t), z(\lambda t), \beta_{z}(t)\right)\right| \\
& \leq \theta_{0}(t)+\theta_{1}(t)(|z(t)|+|z(\lambda t)|)+\theta_{2}\left|\beta_{z}(t)\right| .
\end{aligned}
$$

Taking maximum of both sides and simplifying, we have

$$
\begin{aligned}
\left\|\beta_{z}\right\|_{\mathcal{M}} & \leq \frac{\theta_{0}^{*}+2 \theta_{1}^{*}\|z\|_{\mathcal{M}}}{1-\theta_{2}^{*}} \\
& \leq \frac{\theta_{0}^{*}+2 \theta_{1}^{*} r}{1-\theta_{2}^{*}}=\mu .
\end{aligned}
$$

Using property $\left(\mathscr{P}_{2}\right)$ of the Green's function $\mathcal{W}(t, s)$ given in Lemma 2 and inequality (16) in inequality (15), we obtain

$$
\|\mathcal{N} z\|_{\mathcal{M}} \leq \mu \varpi
$$

which shows that $\mathcal{N}$ is uniformly bounded. To derive equicontinuity of $\mathcal{N}$, let $t_{1}, t_{2} \in[0, T]$ such that $t_{1} \leq t_{2}$, then

$$
\begin{aligned}
\left|\mathcal{N} z\left(t_{2}\right)-\mathcal{N} z\left(t_{1}\right)\right| \leq & \frac{1}{\Gamma(\alpha)} \int_{0}^{t_{1}}\left[\left(t_{1}-s\right)^{\alpha-1}-\left(t_{2}-s\right)^{\alpha-1}\right]\left|f\left(s, z(s), z(\lambda s), \beta_{z}(s)\right)\right| d s \\
& +\int_{t_{1}}^{t_{2}} \frac{\left(t_{2}-s\right)^{\alpha-1}}{\Gamma(\alpha)}\left|f\left(s, z(s), z(\lambda s), \beta_{z}(s)\right)\right| d s \\
& +\frac{\Gamma(2-p)\left(t_{2}-t_{1}\right)}{T^{1-p} \Gamma(\alpha-p)} \int_{0}^{T}(T-s)^{\alpha-p-1}\left|f\left(s, z(s), z(\lambda s), \beta_{z}(s)\right)\right| d s \\
& +\frac{\Gamma(3-q)\left(t_{2}-t_{1}\right) T^{q-1}}{(2-p) \Gamma(\alpha-q)} \int_{0}^{T}(T-s)^{\alpha-q-1}\left|f\left(s, z(s), z(\lambda s), \beta_{z}(s)\right)\right| d s \\
& +\frac{\Gamma(3-q)\left(t_{2}-t_{1}\right)^{2}}{2 \Gamma(\alpha-q) T^{2-q}} \int_{0}^{T}(T-s)^{\alpha-q-1}\left|f\left(s, z(s), z(\lambda s), \beta_{z}(s)\right)\right| d s \\
\leq & \frac{\mu\left(2\left(t_{2}-t_{1}\right)^{\alpha}+\left(t_{2}^{\alpha}-t_{1}^{\alpha}\right)\right)}{\Gamma(\alpha+1)}+\frac{\mu \Gamma(2-p)\left(t_{2}-t_{1}\right) T^{\alpha-1}}{\Gamma(\alpha-p+1)} \\
& +\frac{\mu \Gamma(3-q)\left(t_{2}-t_{1}\right) T^{\alpha-1}}{(2-p) \Gamma(\alpha-q+1)}+\frac{\mu \Gamma(3-q)\left(t_{2}-t_{1}\right)^{2} T^{\alpha-2}}{2 \Gamma(\alpha-q+1)} .
\end{aligned}
$$

From (17), we see that as $t_{1} \rightarrow t_{2}$, the right-hand side tends to zero. Therefore

$$
\left|\mathcal{N} z\left(t_{2}\right)-\mathcal{N} z\left(t_{1}\right)\right| \rightarrow 0, \quad \text { as } t_{1} \rightarrow t_{2}
$$


Since $\mathcal{N}$ is uniformly bounded, so we can also get that

$$
\left\|\mathcal{N} z\left(t_{2}\right)-\mathcal{N} z\left(t_{1}\right)\right\|_{\mathcal{M}} \rightarrow 0, \quad \text { as } t_{1} \rightarrow t_{2},
$$

which confirms the equicontinuity of the operator $\mathcal{N}$. Analogously $\mathcal{N}(\mathscr{B}) \subset \mathscr{B}$. Thus, by Arzelá-Ascoli theorem, $\mathcal{N}$ is completely continuous.

Theorem 3 Under the complete continuity of operator $\mathcal{N}$ and hypotheses $\left(F_{1}\right),\left(F_{2}\right)$, problem (1) has at least one solution.

Proof We define a set $\mathscr{E}$ as

$$
\mathscr{E}=\{z \in \mathcal{M}: z=\rho \mathcal{N}(z), \rho \in(0,1)\} .
$$

The operator $\mathcal{N}: \overline{\mathscr{E}} \rightarrow \mathcal{M}$ as defined in (14) is completely continuous by Theorem 2 . Take $z \in \mathscr{E}$. Then, by definition of the set $\mathscr{E}$ and $\left(F_{2}\right)$, we have

$$
\begin{aligned}
|z(t)| & =|\rho \mathcal{N}(z)(t)| \\
& \leq \rho \max _{t \in[0, T]} \int_{0}^{T}|\mathcal{W}(t, s)|\left|f\left(s, z(s), z(\lambda s), \beta_{z}(s)\right)\right| d s \\
& \leq \max _{t \in[0, T]} \int_{0}^{T}|\mathcal{W}(t, s)| \frac{\theta_{0}^{*}+2 \theta_{1}^{*} r}{1-\theta_{2}^{*}} d s,
\end{aligned}
$$

from which we have

$$
\|z\|_{\mathcal{M}} \leq \varpi \mu .
$$

Hence the set $\mathscr{E}$ is bounded. So the operator $\mathcal{N}$ has at least one solution. Consequently, problem (1) maintains at least one solution.

Theorem 4 If hypothesis $\left(F_{1}\right)$ and the condition $\frac{L_{f}}{1-K_{f}} \varpi<1$ hold, where $\varpi$ is given in (13), then problem (1) has the unique solution in $\mathcal{M}$.

Proof Here we shall use the Banach theorem to prove the required result. Let $z, \bar{z} \in \mathcal{M}$. Then for $t \in[0, T]$ consider

$$
\begin{aligned}
|\mathcal{N} z(t)-\mathcal{N} \bar{z}(t)| & =\left|\int_{0}^{T} \mathcal{W}(t, s)\left(\beta_{z}(s)-\beta_{\bar{z}}(s)\right) d s\right| \\
& \leq \int_{0}^{T}|\mathcal{W}(t, s)|\left|\beta_{z}(s)-\beta_{\bar{z}}(s)\right| d s,
\end{aligned}
$$

where

$$
\begin{aligned}
& \beta_{z}(t)=f\left(t, z(t), z(\lambda t), \beta_{z}(t)\right), \\
& \beta_{\bar{z}}(t)=f\left(t, \bar{z}(t), \bar{z}(\lambda t), \beta_{\bar{z}}(t)\right) .
\end{aligned}
$$


By $\left(F_{1}\right)$, we get

$$
\begin{aligned}
\left|\beta_{z}(t)-\beta_{\bar{z}}(t)\right| & =\left|f\left(t, z(t), z(\lambda t), \beta_{z}(t)\right)-f\left(t, \bar{z}(t), \bar{z}(\lambda t), \beta_{\bar{z}}(t)\right)\right| \\
& \leq L_{f}(|z(t)-\bar{z}(t)|+|z(\lambda t)-\bar{z}(\lambda t)|)+K_{f}\left|\beta_{z}(t)-\beta_{\bar{z}}(t)\right| \\
& \leq \frac{2 L_{f}}{\left(1-K_{f}\right)}|z(t)-\bar{z}(t)|,
\end{aligned}
$$

which implies

$$
\left\|\beta_{z}-\beta_{\bar{z}}\right\| \leq \frac{2 L_{f}}{\left(1-K_{f}\right)}\|z-\bar{z}\| .
$$

Using this result and property $\left(\mathscr{P}_{2}\right)$, from (19), we have

$$
\begin{aligned}
\|\mathcal{N} z-\mathcal{N} \bar{z}\|_{\mathcal{M}} & \leq \max _{t \in[0, T]} \int_{0}^{T}|\mathcal{W}(t, s)| \frac{2 L_{f}}{1-K_{f}}\|z-\bar{z}\|_{\mathcal{M}} d s \\
& \leq \frac{2 L_{f} \varpi}{1-K_{f}}\|z-\bar{z}\|_{\mathcal{M}} .
\end{aligned}
$$

Since $\frac{2 L_{f} \varpi}{1-K_{f}}<1$, the operator $\mathcal{N}$ is contraction, and thus by the Banach contraction theorem, problem (1) has the unique solution.

\section{Stability results}

The present part of our article addresses stability results for the proposed problem.

Lemma 3 For the given problem of pantograph implicit fractional order differential equations with $t \in[0, T]$

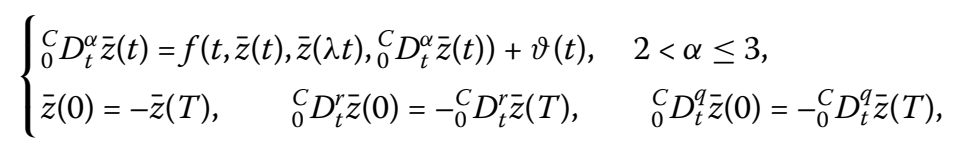

we have the following inequality:

$$
\left|\bar{z}(t)-\int_{0}^{T} \mathcal{W}(t, s) f\left(s, \bar{z}(s), \bar{z}(\lambda s),{ }_{0}^{C} D_{t}^{\alpha} \bar{z}(s)\right) d s\right| \leq \varpi \epsilon,
$$

where $\epsilon>0$.

Proof Thanks to Corollary 1 the solution of perturbed problem (20) is given by

$$
\bar{z}(t)=\int_{0}^{T} \mathcal{W}(t, s) f\left(s, \bar{z}(s), \bar{z}(\lambda s),{ }^{c} D^{\alpha} \bar{z}(s)\right) d s+\int_{0}^{T} \mathcal{W}(t, s) \vartheta(s) d s
$$

from which we have, by using (i) of Remark 1 and property $\left(\mathscr{P}_{2}\right)$ of $\mathcal{W}$,

$$
\begin{aligned}
\left|\bar{z}(t)-\int_{0}^{T} \mathcal{W}(t, s) f\left(s, \bar{z}(s), \bar{z}(\lambda s),{ }^{c} D^{\alpha} \bar{z}(s)\right) d s\right| & \leq \int_{0}^{T}|\mathcal{W}(t, s)||\vartheta(s)| d s \\
& \leq \varpi \epsilon, \quad t \in[0, T] .
\end{aligned}
$$


Theorem 5 If the conditions $K_{f}+2 L_{f} \varpi<1$ and $K_{f}<1$ hold, then the solution of (1) is Hyers-Ulam stable and generalized Hyers-Ulam stable.

Proof Let $z \in \mathcal{M}$ be the unique solution of problem (1) and $\bar{z} \in \mathcal{M}$ be any solution of inequality (21). Then consider

$$
\begin{aligned}
\|\bar{z}-z\|_{\mathcal{M}}= & \max _{t \in[0, T]}\left|\bar{z}-\int_{0}^{T} \mathcal{W}(t, s) f\left(s, z(s), z(\lambda s),{ }_{0}^{C} D_{t}^{\alpha} z(s)\right) d s\right| \\
\leq & \max _{t \in[0, T]}\left|\bar{z}-\int_{0}^{T} \mathcal{W}(t, s) f\left(s, \bar{z}(s), \bar{z}(\lambda s),{ }_{0}^{C} D_{t}^{\alpha} \bar{z}(s)\right) d s\right| \\
& +\max _{t \in[0, T]} \mid \int_{0}^{T} \mathcal{W}(t, s) f\left(s, \bar{z}(s), \bar{z}(\lambda s),{ }_{0}^{C} D_{t}^{\alpha} \bar{z}(s)\right) d s \\
& -\int_{0}^{T} \mathcal{W}(t, s) f\left(s, z(s), z(\lambda s),{ }_{0}^{C} D_{t}^{\alpha} z(s)\right) d s \mid
\end{aligned}
$$

By the application of assumption $\left(F_{1}\right)$ and Lemma 3 , we get

$$
\|\bar{z}-z\|_{\mathcal{M}} \leq \varpi \epsilon+\frac{2 L_{f} \varpi}{1-K_{f}}\|\bar{z}-z\|_{\mathcal{M}}
$$

Upon simplification (22) yields

$$
\|\bar{z}-z\| \leq \mathrm{C}_{f} \epsilon ; \quad \mathrm{C}_{f}=\frac{\varpi\left(1-K_{f}\right)}{1-\left(K_{f}+2 L_{f} \varpi\right)}
$$

Hence problem (1) is Hyers-Ulam stable. Further, if there exists a nondecreasing function $\zeta:(0,1) \rightarrow(0, \infty)$ such that $\zeta(\epsilon)=\epsilon$ with $\zeta(0)=0$, then from (22) we have

$$
\|\bar{z}-z\| \leq \mathrm{C}_{f} \zeta(\epsilon)
$$

Thus problem (1) is generalized Hyers-Ulam stable.

Lemma 4 For the given problem (20), the following inequality holds:

$$
\left|\bar{z}(t)-\int_{0}^{T} \mathcal{W}(t, s) f\left(s, \bar{z}(s), \bar{z}(\lambda s),{ }_{0}^{C} D_{t}^{\alpha} \bar{z}(s)\right) d s\right| \leq \varpi \xi(t) \epsilon, \quad t \in[0, T] .
$$

Proof Thanks to Corollary 1 the solution of the perturbed problem (20) is given by

$$
\bar{z}(t)=\int_{0}^{T} \mathcal{W}(t, s) f\left(s, \bar{z}(s), \bar{z}(\lambda s),{ }_{0}^{C} D_{t}^{\alpha} \bar{z}(s)\right) d s+\int_{0}^{T} \mathcal{W}(t, s) \vartheta(s) d s .
$$

Using (i) of Remark 2 and property $\left(\mathscr{P}_{2}\right)$, we have

$$
\begin{aligned}
\left|\bar{z}(t)-\int_{0}^{T} \mathcal{W}(t, s) f\left(s, \bar{z}(s), \bar{z}(\lambda s),{ }_{0}^{C} D_{t}^{\alpha} \bar{z}(s)\right) d s\right| & \leq \int_{0}^{T}|\mathcal{W}(t, s)||\vartheta(s)| d s \\
& \leq \varpi \xi(t) \epsilon, \quad t \in[0, T]
\end{aligned}
$$


Theorem 6 If hypothesis $\left(F_{1}\right)$ together with the conditions $K_{f}+2 L_{f} \varpi<1, K_{f}<1$ holds, then problem (1) is Hyers-Ulam-Rassias stable.

Proof Let $\bar{z}$ be any solution of inequality (25) and $z \in \mathcal{M}$ be the unique solution of problem (1). Then

$$
\begin{aligned}
\|\bar{z}-z\|_{\mathcal{M}}= & \max _{t \in[0, T]}\left|\bar{z}-\int_{0}^{T} \mathcal{W}(t, s) f\left(s, z(s), z(\lambda s),{ }_{0}^{C} D_{t}^{\alpha} z(s)\right) d s\right| \\
\leq & \max _{t \in[0, T]}\left|\bar{z}-\int_{0}^{T} \mathcal{W}(t, s) f\left(s, \bar{z}(s), \bar{z}(\lambda s),{ }_{0}^{C} D_{t}^{\alpha} \bar{z}(s)\right) d s\right| \\
& +\max _{t \in[0, T]} \mid \int_{0}^{T} \mathcal{W}(t, s) f\left(s, \bar{z}(s), \bar{z}(\lambda s),{ }_{0}^{C} D_{t}^{\alpha} \bar{z}(s)\right) d s \\
& -\int_{0}^{T} \mathcal{W}(t, s) f\left(s, z(s), z(\lambda s),{ }_{0}^{C} D_{t}^{\alpha} z(s)\right) d s \mid .
\end{aligned}
$$

By the application of assumption $\left(F_{1}\right)$ and Lemma 4 , we get

$$
\|\bar{z}-z\|_{\mathcal{M}} \leq \varpi \xi(t) \epsilon+\frac{2 L_{f} \varpi}{1-K_{f}}\|\bar{z}-z\|_{\mathcal{M}}
$$

Upon simplification (26) gives

$$
\|\bar{z}-z\|_{\mathcal{M}} \leq \mathrm{C}_{f} \xi(t) \epsilon, \quad \mathrm{C}_{f}=\frac{\varpi\left(1-K_{f}\right)}{1-\left(K_{f}+2 L_{f} \varpi\right)} .
$$

Thus problem (1) is Hyers-Ulam-Rassias stable.

Lemma 5 The solution of the perturbed problem given in (20) produces the given relation

$$
\left|\bar{z}(t)-\int_{0}^{T} \mathcal{W}(t, s) f\left(s, \bar{z}(s), \bar{z}(\lambda s),{ }_{0}^{C} D_{t}^{\alpha} \bar{z}(s)\right) d s\right| \leq \varpi \xi(t), \quad t \in[0, T] .
$$

Proof For the proof, follow Lemma 3.

Theorem 7 Under hypothesis $\left(F_{1}\right)$ and the inequalities $K_{f}+2 L_{f} \varpi<1, K_{f}<1$, the solution of problem (1) is generalized Hyers-Ulam-Rassias stable.

Proof Just like Theorem 6, we have

$$
\|\bar{z}-z\|_{\mathcal{M}} \leq \mathrm{C}_{f} \xi(t), \quad \mathrm{C}_{f}=\frac{\varpi\left(1-K_{f}\right)}{1-\left(K_{f}+2 L_{f} \varpi\right)}
$$

Hence problem (1) is generalized Hyers-Ulam-Rassias stable.

\section{Test problems}

To test our theoretical results, we present some problems here. 
Problem 1 Consider the following problem of pantograph implicit fractional order differential equations with given anti-periodic boundary conditions:

$$
\left\{\begin{array}{l}
{ }_{0}^{C} D_{t}^{\frac{5}{2}} z(t)=\frac{1}{150}\left[t \cos z(t)-z\left(\frac{1}{7} t\right) \sin (t)\right]+\frac{{ }_{0}^{C} D_{t}{ }^{\frac{5}{2}} z(t)}{{ }_{100+}{ }_{0}^{C} D_{t}^{\frac{5}{2}} z(t)}, \quad t \in[0, T]=[0,1], \\
z(0)=-z(1), \quad{ }_{0}^{C} D_{t}^{\frac{1}{2}} z(0)=-{ }_{0}^{C} D_{t}^{\frac{1}{2}} z(1), \quad{ }_{0}^{C} D_{t}^{\frac{3}{2}} z(0)=-{ }_{0}^{C} D_{t}^{\frac{3}{2}} z(1) .
\end{array}\right.
$$

Here,

$$
\begin{aligned}
f\left(t, z(t), z(\lambda t),{ }_{0}^{C} D_{t}^{\alpha} z(t)\right)= & \frac{1}{150}\left[t \cos z(t)-z\left(\frac{1}{7} t\right) \sin (t)\right] \\
& +\frac{{ }_{0}^{C} D_{t}^{\frac{5}{2}} z(t)}{100+{ }_{0}^{C} D_{t}^{\frac{5}{2}} z(t)}
\end{aligned}
$$

with $\alpha=\frac{5}{2}, p=\frac{1}{2}, q=\frac{3}{2}, \lambda=\frac{1}{7}, T=1$. The continuity of $f$ is obvious.

By hypothesis $\left(F_{1}\right)$, for any $z, \bar{z} \in R$, we have

$$
\begin{aligned}
\left|f\left(t, z(t), z(\lambda t),{ }_{0}^{C} D_{t}^{\alpha} z(t)\right)-f\left(t, \bar{z}(t), \bar{z}(\lambda t),{ }_{0}^{C} D_{t}^{\alpha} \bar{z}(t)\right)\right| \leq & \frac{1}{150}[|z(t)-\bar{z}(t)|+|z(t)-\bar{z}(t)|] \\
& +\frac{1}{100}\left|{ }_{0}^{C} D_{t}^{\frac{5}{2}} z(t)-{ }_{0}^{C} D_{t}^{\frac{5}{2}} \bar{z}(t)\right| \\
= & \frac{1}{150}[2|z(t)-\bar{z}(t)|] \\
& +\frac{1}{100}\left|{ }_{0}^{C} D_{t}^{\frac{5}{2}} z(t)-{ }_{0}^{C} D_{t}^{\frac{5}{2}} \bar{z}(t)\right| .
\end{aligned}
$$

Hence we have $L_{f}=\frac{1}{150}, K_{f}=\frac{1}{100}$. On computation, we have $\varpi=1.26098028$. Now, thanks to Theorem 4, we see that

$$
\frac{2 L_{f} \varpi}{1-K_{f}}=1.6982 \times 10^{-2}<1
$$

Thus the given problem (1) has at most one solution. Further, on using Theorem 5, we see that

$$
K_{f}+2 L_{f} \varpi=0.0168130+0.01=0.0268130704<1 .
$$

Hence the solution is Hyers-Ulam stable. Further, it is also generalized Hyers-Ulam stable. For Hyers-Ulam-Rassias stability, we apply Theorem 6 by taking a nondecreasing function $\xi(t)=t$ for $t \in(0,1)$. One has $C_{f}=\frac{\varpi\left(1-K_{f}\right)}{1-\left(K_{f}+2 L_{f} \varpi\right)}=1.2828$. Hence we see that, for the unique solution $\bar{z} \in \mathcal{M}$ and any solution $z \in \mathcal{M}$, the following relation holds true:

$$
\|z-\bar{z}\|_{\mathcal{M}} \leq 1.2828 \epsilon t \quad \text { for all } t \in[0,1] .
$$

Hence the solution of (1) is Hyers-Ulam-Rassias stable. Consequently, it is obviously generalized Hyers-Ulam-Rassias stable on using Theorem 7. 
Problem 2 Here we take another problem of pantograph implicit fractional order differential equations

$$
\left\{\begin{array}{l}
{ }_{0}^{C} D_{t}^{\frac{5}{2}} z(t)=\frac{e^{-\pi t}}{10}+\frac{e^{-t}}{40+t^{2}}\left(\sin (|z(t)|)+z\left(\frac{1}{4} t\right)+\sin \left(\left|{ }_{0}^{C} D_{t}^{\frac{5}{2}} z(t)\right|\right)\right), \quad t \in[0,1], \\
z(0)=-z(1), \quad{ }_{0}^{C} D_{t}^{\frac{1}{2}} z(0)=-{ }_{0}^{C} D_{t}^{\frac{1}{2}} z(1), \quad{ }_{0}^{C} D_{t}^{\frac{3}{2}} z(0)=-{ }_{0}^{C} D_{t}^{\frac{3}{2}} z(1) .
\end{array}\right.
$$

Here,

$$
\begin{aligned}
f\left(t, z(t), z(\lambda t),{ }_{0}^{C} D_{t}^{\alpha} z(t)\right)= & \frac{\exp (-\pi t)}{10}+\frac{\exp (-t)}{40+t^{2}} \sin (|z(t)|)+z\left(\frac{1}{4} t\right) \\
& +\frac{\exp (-t)}{40+t^{2}} \sin \left(\left|{ }_{0}^{C} D_{t}^{\frac{5}{2}} z(t)\right|\right),
\end{aligned}
$$

with $\alpha=\frac{5}{2}, p=\frac{1}{2}, q=\frac{3}{2}, \lambda=\frac{1}{4}, T=1$. The continuity of $f$ is obvious.

Now, for any $z, \bar{z} \in \mathcal{M}$, and $t \in[0,1]$, we have

$$
\begin{aligned}
\left|f\left(t, z(t), z(\lambda t),{ }_{0}^{C} D_{t}^{\alpha} z(t)\right)-f\left(t, \bar{z}(t), \bar{z}(\lambda t),{ }_{0}^{C} D_{t}^{\alpha} \bar{z}(t)\right)\right| \leq & \frac{1}{40}[2|z(t)-\bar{z}(t)| \\
& \left.+\left|{ }_{0}^{C} D_{t}{ }^{\frac{5}{2}} z(t)-{ }_{0}^{C} D_{t}^{\frac{5}{2}} \bar{z}(t)\right|\right] .
\end{aligned}
$$

Hence $f$ satisfies hypothesis $\left(F_{1}\right)$ with $L_{f}=K_{f}=\frac{1}{40}$. The function $f$ also satisfies hypothesis $\left(F_{2}\right)$ with $\theta_{0}(t)=\frac{\exp (-\pi t)}{10}, \theta_{1}(t)=\theta_{2}(t)=\frac{\exp (-t)}{40+t}$, where $\theta_{0}^{*}(t)=\frac{1}{10}, \theta_{1}^{*}(t)=\theta_{2}^{*}(t)=\frac{1}{40}$. Upon calculation, we get

$$
\begin{aligned}
\varpi & =\frac{1}{\Gamma\left(\frac{5}{2}+1\right)}+\frac{\Gamma\left(2-\frac{1}{2}\right)}{2 \Gamma\left(\frac{5}{2}-\frac{1}{2}+1\right)}+\frac{\left(\frac{1}{2}+2\right)\left(\Gamma\left(3-\frac{3}{2}\right)\right)}{2\left(2-\frac{1}{2}\right) \Gamma\left(\frac{5}{2}-\frac{3}{2}+1\right)} \\
& =1.26098028 .
\end{aligned}
$$

Thanks to Theorem 3, we see that $\mu=\frac{\theta_{0}^{*} \varpi}{1-\left(\theta_{2}^{*}+2 \theta_{1}^{*} \varpi\right)}=0.1383$, and therefore the condition $\theta_{2}^{*}+2 \theta_{1}^{*} \varpi<1$ holds true. Thus the given problem (2) has at least one solution. Further, using Theorem 4 , we see that

$$
\frac{2 L_{f} \varpi}{1-K_{f}}=6.46656 \times 10^{-2}<1 .
$$

So the criteria for unique solution have been followed. Further, by using Theorem 5, we observe that

$$
K_{f}+2 L_{f} \varpi=11.3048 \times 10^{-2}<1
$$

Hence the solution is Hyers-Ulam stable. Further, it is also generalized Hyers-Ulam stable. For Hyers-Ulam-Rassias stability, we use our Theorem 6 by taking a nondecreasing function $\xi(t)=t$ for $t \in(0,1)$. One has $C_{f}=\frac{\varpi\left(1-K_{f}\right)}{1-\left(K_{f}+2 L_{f} \varpi\right)}=1.38616$. Hence we see that, for any solution $\bar{z} \in \mathcal{M}$ and the unique solution $z \in \mathcal{M}$, the following relation holds true:

$$
\|\bar{z}-z\|_{\mathcal{M}} \leq 1.38616 \epsilon t \quad \text { for all } t \in[0,1] .
$$


Hence the solution of (1) is Hyers-Ulam-Rassias stable. Consequently, it is obviously generalized Hyers-Ulam-Rassias stable on using Theorem 7.

Problem 3 Consider the third example of pantograph implicit fractional order differential equations:

$$
\left\{\begin{array}{l}
{ }_{0}^{C} D_{t}^{\frac{7}{3}} z(t)=\frac{t}{50}+\frac{\left(t^{2}+3\right)}{40} \sqrt{|z(t)|}+\frac{\left(t^{2}+3\right)}{40} \sqrt{\left|z\left(\frac{1}{5} t\right)\right|} \\
\quad+\frac{\left(t^{2}+3\right)}{40}{ }_{0}^{C} D_{t}^{\frac{7}{3}}(z(t)), \quad t \in[0,2], \\
z(0)=-z(2), \quad{ }_{0}^{C} D_{t}^{\frac{1}{3}} z(0)=-{ }_{0}^{C} D_{t}^{\frac{1}{3}} z(2), \quad{ }_{0}^{C} D_{t}^{\frac{4}{3}} z(0)=-{ }_{0}^{C} D_{t}^{\frac{4}{3}} z(2) .
\end{array}\right.
$$

Here,

$$
\begin{aligned}
f\left(t, z(t), z(\lambda t),{ }_{0}^{C} D_{t}^{\alpha} z(t)\right)= & \frac{t}{50}+\frac{\left(t^{2}+3\right)}{40} \sqrt{|z(t)|}+\frac{\left(t^{2}+3\right)}{40} \sqrt{\left|z\left(\frac{1}{5} t\right)\right|} \\
& +\frac{\left(t^{2}+3\right)}{40}{ }_{0}^{C} D_{t}^{\frac{7}{3}}(z(t))
\end{aligned}
$$

with $\alpha=\frac{7}{3}, p=\frac{1}{3}, q=\frac{4}{3}, T=2$. For any $z, \bar{z} \in R$ and $t \in[0,2]$, let us have

$$
\begin{aligned}
\left|f\left(t, z(t), z(\lambda t),{ }_{0}^{C} D_{t}^{\alpha} z(t)\right)-f\left(t, \bar{z}(t), \bar{z}(\lambda t),{ }_{0}^{C} D_{t}^{\alpha} \bar{z}(t)\right)\right| \leq & \frac{1}{9}[2|z(t)-\bar{z}(t)| \\
& \left.+\left|{ }_{0}^{C} D_{t}{ }^{\frac{7}{3}} z(t)-{ }_{0}^{C} D_{t}{ }^{\frac{7}{3}} \bar{z}(t)\right|\right] .
\end{aligned}
$$

Hence $f$ satisfies hypothesis $\left(F_{1}\right)$ with $L_{f}=K_{f}=\frac{1}{9}$. The function $f$ also satisfies hypothesis $\left(F_{2}\right)$ with $\theta_{0}(t)=\frac{t}{50}, \theta_{1}(t)=\theta_{2}(t)=\frac{t^{2}+1}{45}$, where $\theta_{0}^{*}(t)=\frac{1}{25}, \theta_{1}^{*}(t)=\theta_{2}^{*}(t)=\frac{1}{9}$. Upon computation, we can arrive at $\theta_{2}^{*}+\theta_{1}^{*} \varpi<1$ and $\frac{2 L_{f}}{1-K_{f}} \varpi<1$. Thus, on using Theorem 4 , the required results are followed. Moreover, it also satisfies the condition of Hyers-Ulam stability and generalized Hyers-Ulam stability by computing $K_{f}+2 L_{f} \varpi<1$ and using Theorem 5 . Taking a nondecreasing function $\xi(t)=1+t$, problem (3) is Hyers-Ulam-Rassias stable and hence generalized Hyers-Ulam-Rassias stable upon the application of Theorem 6 and Theorem 7 respectively.

\section{Conclusion}

In the present work we have established qualitative analysis of existence results regarding the solution of nonlinear pantograph implicit fractional order differential equations subject to anti-periodic boundary conditions. The respective analysis has been carried out via fixed point theory. Further some adequate results were also developed corresponding to Hyers-Ulam type stability and its various forms. To testify the established theory, some test problems were given in the last section. We concluded that nonlinear analysis is a powerful tool to study applied problems.

\section{Acknowledgements}

We are really thankful to the reviewers for their useful suggestions and corrections. The third author would like to thank Prince Sultan University for the support through the research group Nonlinear Analysis Methods in Applied Mathematics (NAMAM), group number RG-DES-2017-01-17. 
Availability of data and materials

Data sharing not applicable to this article as no datasets were generated or analysed during the current study.

\section{Competing interests}

The authors declare that they have no competing interests.

\section{Authors' contributions}

All authors equally contributed to this manuscript and read and approved the final version.

\section{Author details}

'Department of Mathematics, University of Malakand, Khyber Pakhtunkhwa, Pakistan. ${ }^{2}$ Department of Mathematics and General Sciences, Prince Sultan University, Riyadh, Saudi Arabia. ${ }^{3}$ Department of Medical Research, China Medical University, Taichung, Taiwan. ${ }^{4}$ Department of Computer Science and Information Engineering, Asia University, Taichung, Taiwan.

\section{Publisher's Note}

Springer Nature remains neutral with regard to jurisdictional claims in published maps and institutional affiliations.

Received: 23 November 2019 Accepted: 20 March 2020 Published online: 30 March 2020

\section{References}

1. Kilbas, A.A., Srivastava, H., Trujillo, J.: Theory and Application of Fractional Differential Equations. North-Holland Mathematics Studies, vol. 204. Elsevier, Amsterdam (2006)

2. Hilfer, R.: Applications of Fractional Calculus in Physics. World Scientific, Singapore (2000)

3. Abdeljawad, T., Al-Mdallal, Q.M., Jarad, F.: Fractional logistic models in the frame of fractional operators generated by conformable derivatives. Chaos Solitons Fractals 119, 94-101 (2019)

4. Ren, X., Wang, G., Bai, Z., El-Deeb, A.A.: Maximum principle and its application to multi-index Hadamard fractional diffusion equation. Bound. Value Probl. 2019(1), 182 (2019)

5. Wang, D., Wang, G.: Integro-differential fractional boundary value problem on an unbounded domain. Adv. Differ. Equ. 2016(1), 325 (2016)

6. Wang, G., Cabada, A., Zhang, L.: An integral boundary value problem for nonlinear differential equations of fractional order on an unbounded domain. J. Integral Equ. Appl. 26, 117-129 (2014)

7. Wang, G., Ren, X., Bai, Z., Hou, W.: Radial symmetry of standing waves for nonlinear fractional Hardy-Schrödinger equation. Appl. Math. Lett. 96, 131-137 (2019)

8. Wang, G.: Twin iterative positive solutions of fractional $q$-difference Schrödinger equations. Appl. Math. Lett. 76 103-109 (2018)

9. Zhang, L., Hou, W.: Standing waves of nonlinear fractional $p$-Laplacian Schrödinger equation involving logarithmic nonlinearity. Appl. Math. Lett. 102, 106149 (2020)

10. Podlubny, I.: Fractional Differential Equations. Mathematics in Science and Engineering. Academic Press, New York (1999)

11. Abdeljawad, T.: On Riemann and Caputo fractional differences. Comput. Math. Appl. 62(3), 1602-1611 (2011)

12. Ali, A., Shah, K., Jarad, F., Gupta, V., Abdeljawad, T.: Existence and stability analysis to a coupled system of implicit type impulsive boundary value problems of fractional-order differential equations. Adv. Differ. Equ. 2019, 101 (2019)

13. Shah, K., Kumam, P., Ullah, I.: On Ulam stability and multiplicity results to a nonlinear coupled system with integral boundary conditions. Mathematics 7(3), 223 (2019)

14. Abdeljawad, T., Madjidi, F., Jarad, F., Sene, N.: On dynamic systems in the frame of singular function dependent kernel fractional derivatives. Mathematics 7, 946 (2019)

15. Hashim, I., Abdulaziz, O., Momani, S.: Homotopy analysis method for fractional IVPs. Commun. Nonlinear Sci. Numer. Simul. 14, 674-684 (2009)

16. Coronel-Escamilla, A., Gómez-Aguilar, J.F.: A novel predictor-corrector scheme for solving variable-order fractional delay differential equations involving operators with Mittag-Leffler kernel. Discrete Contin. Dyn. Syst., Ser. S 13(3), 561-574 (2020)

17. Ali, A.: Ulam type stability analysis of implicit impulsive fractional differential equations. M.Phil. dissertation, University of Malakand, Pakistan (2017)

18. Shah, K.: Multipoint boundary value problems for systems of fractional differential equations: existence theory and numerical simulations. Ph.D. dissertation, University of Malakand, Pakistan (2016)

19. Sabri, A.: An analysis of exponential stability of delayed neural networks with time varying delays. Neural Netw. 17(7), 1027-1031 (2004)

20. Jamal, D., Riedinger, P., lung, C.: Stability analysis and control synthesis for switched systems: a switched Lyapunov function approach. IEEE Trans. Autom. Control 47(11), 1883-1887 (2002)

21. Yan, L., Chen, Y., Podlubny, I.: Stability of fractional-order nonlinear dynamic systems: Lyapunov direct method and generalized Mittag-Leffler stability. Comput. Math. Appl. 59(5), 1810-1821 (2010)

22. Hyers, D.H.: On the stability of the linear functional equations. Proc. Natl. Acad. Sci. USA 27(4), 222-224 (1941)

23. Ulam, S.M.: A Collection of the Mathematical Problems. Interscience, New York (1960)

24. Rassias, T.M.: On the stability of the linear mapping in Banach spaces. Proc. Am. Math. Soc. 72(2), 297-300 (1978)

25. Jung, S.M.: Hyers-Ulam stability of linear differential equations of first order. Appl. Math. Lett. 17(10), 1135-1140 (2004)

26. Rus, I.A.: Ulam stabilities of ordinary differential equations in a Banach space. Carpath. J. Math. 26, 103-107 (2010)

27. Wang, J., LV, L., Zhou, W.: Ulam stability and data dependence for fractional differential equations with Caputo derivative. Electron. J. Qual. Theory Differ. Equ. 2011, 63 (2011) 
28. Mendes, E.M., Salgado, G.H., Aguirre, L.A.: Numerical solution of Caputo fractional differential equations with infinity memory effect at initial condition. Commun. Nonlinear Sci. Numer. Simul. 69, 237-247 (2019)

29. Yu, Z.H.: Variational iteration method for solving the multi-pantograph delay equation. Phys. Lett. A 372(43), 6475-6479 (2008)

30. Tohidi, E., Bhrawy, A.H., Erfani, K.: A collocation method based on Bernoulli operational matrix for numerical solution of generalized pantograph equation. Appl. Math. Model. 37(6), 4283-4294 (2013)

31. Balachandran, K., Kiruthika, S., Trujillo, J.J. Existence of solution of nonlinear fractional pantograph equations. Acta Math. Sci. 33(3), 712-720 (2013)

32. Iqbal, M., Shah, K., Khan, R.A.: On using coupled fixed point theorems for mild solutions to coupled system of multi-point boundary value problems of nonlinear fractional hybrid pantograph differential equations. Math. Methods Appl. Sci., 1-14 (2019)

33. Agarwal, R.P., Benchohra, M., Hamani, S.: A survey on existence results for boundary value problems of nonlinear fractional differential equations and inclusions. Acta Appl. Math. 109(3), 973-1033 (2010)

34. Cabada, A., Wang, G.: Positive solutions of nonlinear fractional differential equations with integral boundary value conditions. J. Math. Anal. Appl. 389(1), 403-411 (2013)

35. Ali, Z., Zada, A., Shah, K.: On Ulam's stability for a coupled systems of nonlinear implicit fractional differential equations. Bull. Malays. Math. Sci. Soc. 42(5), 2681-2699 (2018)

\section{Submit your manuscript to a SpringerOpen ${ }^{\circ}$ journal and benefit from:}

- Convenient online submission

- Rigorous peer review

- Open access: articles freely available online

- High visibility within the field

- Retaining the copyright to your article 